\title{
Allergological Study of 565 Elderly Patients Previously Labeled as Allergic to Penicillins
}

This article was published in the following Dove Press journal:

Journal of Asthma and Allergy

Teodorikez Wilfox Jimenez-

Rodriguez (D) ${ }^{\text {I-3 }}$

Natalia Blanca-Lopez $\mathbb{D}^{2,4}$

Maria Ruano-Zaragoza (iD) ${ }^{1-3}$

Victor Soriano-Gomis ${ }^{1,2,5}$

Angel Esteban-Rodriguez ${ }^{6}$

Geronima Riera-Sendra ${ }^{7}$

Antonio Palazon-Bru ${ }^{5}$

Miguel Blanca ${ }^{2,4}$

Jose Manuel Ramos-

Rincon (iD ${ }^{5,8, *}$

Javier Fernandez-

Sanchez $\mathbb{D}^{1,2,5, *}$

'Allergy Section, Alicante General University Hospital-ISABIAL, Alicante, Spain; ${ }^{2}$ ARADyAL Spanish Network, Madrid, Spain; ${ }^{3} \mathrm{PhD}$ Program in Public Health, Medical and Surgical Sciences, Miguel Hernandez University, Alicante, Spain; ${ }^{4}$ Allergy Section, Infanta Leonor University Hospital, Madrid, Spain;

${ }^{5}$ Clinical Medicine Department, Miguel Hernandez University, Alicante, Spain; ${ }^{6}$ Clinical Analysis Department, Alicante General University Hospital, Alicante, Spain; ${ }^{7}$ Hospital Pharmacy Department, Alicante General University Hospital, Alicante, Spain; ${ }^{8}$ Internal Medicine Department, Alicante General University Hospital, Alicante, Spain

*These authors contributed equally to this work

Correspondence: Teodorikez Wilfox Jimenez-Rodriguez

Seccion de Alergologia, Hospital General Universitario de Alicante, Calle Pintor

Baeza I2, Planta 8, Alicante 03010, Spain

Email teodorikez@gmail.com
Purpose: Elderly people thought to have an allergy to beta-lactams (BLs) may tolerate the drugs in subsequent exposures due to initial false labeling of allergies, the spontaneous loss of sensitivity to BLs over time or age-related decline in sensitization. As a result, they may be treated with less appropriate antibiotics, causing more side effects and entailing increased costs for health systems. The aim of this investigation was to assess whether patients in the third and fourth age with previously confirmed allergies to BLs had lost sensitization and could tolerate these antibiotics.

Patients and methods: Patients allergic to BLs were divided into group A (aged 60-79 years) and $\mathrm{B}$ (aged $\geq 80$ years). Clinical history, skin testing, drug challenge tests (DCT) and evaluation of resensitization were used to classify participants as showing immediate reactions, non-immediate reactions, or tolerance. We compared clinical entities, drugs involved, and final outcome by age group.

Results: Of 1362 cases evaluated, 565 underwent an allergological study. The skin was the most common organ involved. Anaphylaxis and side chain reactions were more frequent in group A $(\mathrm{p}<0.01)$, as were positive DCT. Classical benzylpenicillin determinants (benzylpenicilloyl and/or minor determinant mixture) were more frequent triggers in group $\mathrm{B}$ $(p<0.01)$. Resensitization after challenge occurred in very few participants.

Conclusion: The risk for allergy to BLs decreases with age and a history of anaphylaxis by BLs is a predictor of positive results in skin tests (ST). Both immunoglobin E (IgE) and T-cell-mediated responses can disappear in elderly people, who can develop tolerance to these antibiotics. These results are of clinical relevance to patients who need to be treated with antibiotics from this family.

Keywords: drug allergy, beta-lactams, cephalosporins, cross-reactivity, aging, immunosenescence, diagnosis

\section{Introduction}

BLs antibiotics comprise five major families: penicillins, cephalosporins, monobactams, carbapenems, and clavams. ${ }^{1}$ These drugs are the most frequently involved in reactions IgE-mediated, with an estimated prevalence of $5 \%$ to $10 \%$ in the general population. ${ }^{2,3}$

More than $95 \%$ of the people who report allergy to BLs have good tolerance. ${ }^{2,4-6}$ This apparent contradiction may have different explanations: drug side effects that are not by themselves allergic in nature, ${ }^{4,7,8}$ the attribution of the allergic reaction to these antibiotics when they are caused by other drugs, ${ }^{9}$ or the spontaneous loss of sensitivity to BLs over time. ${ }^{10,11}$

An incorrect diagnosis of BL allergy may lead to the prescription of second-line antibiotics, which can be more toxic, be related to the development of multi-resistant bacterial strains $^{2,12-15}$ and to the increase in costs. ${ }^{7,8,13,16,17}$ 
One problem of major concern is antibiotic prescription in the elderly, because of their immunological decline,${ }^{18}$ this group is more susceptible to systemic infections. Whether this decline has an impact on the $\operatorname{IgE}$ system is unconfirmed, but it is reasonable to assume that this decline may not only affect the $\operatorname{IgG}, \operatorname{IgA}$, and IgM antibody response. ${ }^{18,19}$

Preliminary evidence from our group indicates that older people have a lower prevalence of BL allergy than the general population. ${ }^{20}$ Based on these observations, our aim was to estimate the prevalence before and after an allergological work-up in elderly patients who were referred to our centers for a study on BL allergy after having been previously diagnosed as allergic in different medical services including Geriatrics, Internal Medicine, and Family Medicine.

In this study, we tested the hypothesis that impaired IgE response could be another mechanism for tolerance after drug re-exposure and proved that allergy to BLs decreases with age, probably because of both humoral and cellular immunosenescence.

\section{Materials and Methods}

\section{Patients}

We studied patients aged 60 years or older with a history of hypersensitivity reactions (HSRs) to BLs, who were admitted in the Internal Medicine Service or the Infectious Diseases Unit, or attended for evaluation in the Allergy Service of the Alicante University General Hospital (Alicante, Spain) and Infanta Leonor University Hospital (Madrid, Spain) from November 2015 to November 2017. Patients were classified by age into two groups, according to cutoffs reported elsewhere: ${ }^{21}$ group A, 60 to 79 years; and group B, 80 years or older.

\section{Strategy for Recruitment}

Cases included in the study were recruited from two groups:

1. Patients admitted in the participating services and either previously labeled as allergic to BL or labeled as having HSR to BLs during the hospital stay.

2. Patients with a history of drug HSR to BLs and referred to the outpatient allergy clinic for evaluation.

\section{Clinical History}

We undertook a detailed clinical history following the guidelines of the expert group of the European Academy of Allergy and Clinical Immunology (EAACI). ${ }^{22}$ We also considered the time interval between drug intake and onset of symptoms, duration of treatment before the reaction occurred, and response to treatment.

\section{Classification of Reactions}

Following the EAACI guidelines, immediate hypersensitivity reactions (IHSR) were defined as those that appeared within 20 mins of drug intake and lasted no longer than 1 hr. Involvement of two or more organs was classified as anaphylaxis. $^{23}$

Non-immediate hypersensitivity reactions (NIHR) usually appear at about 24 to $48 \mathrm{hrs}$ but may present as early as $1 \mathrm{hr}$ after drug intake. Limited organ-specific reactions like hepatitis, renal failure, pneumonitis, anemia, neutropenia, and thrombocytopenia were not included in our study. ${ }^{24}$

Before undertaking the study, all patients and/or their authorized representatives were informed of the potential risks and benefits of skin testing and DCT, and they signed written informed consent to participate. The study conforms to the standards of the Declaration of Helsinki. The protocol was approved by the Hospital Ethics Committee (CEIC PI2014/53).

\section{Skin Testing}

ST began by prick test that was considered positive if a wheal greater than $3 \mathrm{~mm}$ appeared surrounded by erythema within 20 mins of application. If negative, we proceeded to intradermal testing with $0.02 \mathrm{~mL}$ of $\mathrm{BL}$ solution, repeating the reading at the same time interval. Different 10 -fold dilutions were used in cases with severe symptoms. Reactions were considered positive if the initial injection papule increased in size by $3 \mathrm{~mm}$ or more. ${ }^{25,26} \mathrm{In}$ case of a clinical history indicative of a NIHSR, a reading took place at 24 and $48 \mathrm{hrs}$ after skin testing. In all cases, we advised patients to monitor the delayed response for upto 1 week after testing.

\section{Reagents for Skin Testing}

ST was carried out in the forearm as previously reported ${ }^{25-28}$ with the following penicillin allergenic determinants: benzylpenicilloyl-poly-L-lysine (BPO-PPL $5 \times 10^{-5} \mathrm{M}$ ) and minor determinant mixture (MDM $2 \times 10^{-2} \mathrm{M}$ ) (provided by Diater, Madrid, Spain); penicillin G $(10,000 \mathrm{IU} / \mathrm{mL}$ : ERN Laboratories); ampicillin (20mg/mL: Normon Laboratories); amoxicillin (20mg/mL: GSK Laboratories); amoxicillin/clavulanic acid (20mg/mL: Normon Laboratories); cefazolin $(20 \mathrm{mg} / \mathrm{mL}$ : Normon Laboratories); cefotaxime (20 $\mathrm{mg} / \mathrm{mL}$ : Normon 
Laboratories); and meropenem (20mg/mL: Kern Pharma Laboratories). In addition, clavulanic acid $(5 \mathrm{mg} / \mathrm{mL}$ and 20mg/mL: Diater, Madrid, Spain); ceftriaxone $(20 \mathrm{mg} / \mathrm{mL}$ : Normon Laboratories); cefuroxime $(20 \mathrm{mg} / \mathrm{mL}$ : Normon Laboratories); imipenem/cilastatin (20mg/mL: Pfizer, S.L.); piperacillin/tazobactam (20mg/mL: Mylan); and cloxacillin (20mg/mL: Normon Laboratories) were also tested when one of them was the culprit drug.

The specificity of the drugs included above was assessed by testing a matched control group with previously established good tolerance. These concentrations proved to be non-irritant in a group of 20 patients with similar age and clinical entities as study participants.

For the non-commercially available diagnosis reagents, testing solutions were prepared by the hospital pharmacy service by diluting them in saline under sterile conditions according to the manufacturer's recommendations. Each vial was used on the same day it was prepared.

\section{Serum Collection}

Venipuncture was used to extract $10 \mathrm{~mL}$ of blood for sera. All samples were maintained at $-80^{\circ} \mathrm{C}$ until analyzed.

\section{In vitro Total IgE and Specific IgE (slgE) Antibodies}

Total IgE and sIgE to penicilloyl G, penicilloyl V, ampicilloyl, amoxicilloyl, and cefaclor were determined using commercially available immunoassays following the manufacturer's instructions (Elecsys IgE II Immunoassay, Roche Diagnostics, USA and ImmunoCAP, Thermo Fisher Scientific, USA, respectively), values of total $\mathrm{IgE}$ exceeding $100 \mathrm{IU} / \mathrm{mL}$ were considered high and $\operatorname{sIgE}>0.35$ $\mathrm{kU} / \mathrm{L}$ were considered positive.

\section{Drug Challenge Test}

The performance of DCT was decided based on the clinical history and ST results. In patients with medical conditions that limited the oral route, the challenge used the intravenous one, as detailed below.

According to the culprit drug and treatment needs, DCTs were done with penicillin $\mathrm{V}$, amoxicillin, ampicillin, amoxicillin/clavulanic acid, cloxacillin, cefditoren, cefuroxime or meropenem. In those experiencing a reaction with benzyl-penicillin, we performed the DCT with amoxicillin unless the patient needed to be treated with it, as we considered that amoxicillin is the most commonly used $\mathrm{BL}$ and is valid to identify BPO-cross reactions and specific side chain reactions. $^{29}$

In patients who reacted to other BLs, after obtaining negative results in the $\mathrm{ST}$ with the determinants described above, we proceeded with skin testing of the culprit drug. If this result was also negative, we undertook DCT.

In patients with a clinical history suggesting an IgEmediated response, a single-blind placebo-controlled DCT (oral or intravenous) was performed, starting with 1/100 of the therapeutic dose, followed by a 10 -fold increase in dose until reaching the cumulative dose of $500 \mathrm{mg}$. In patients with good tolerance, the diagnosis of allergy was ruled out.

If the clinical history was suggestive of a NIHSR, a negative skin test was followed by a single oral dose of $100 \mathrm{mg}$. If no response appeared within $48 \mathrm{hrs}$, we proceeded with the full therapeutic dose, as described previously. ${ }^{30}$ If the DCT was negative, treatment with therapeutic doses of the same medication was administered to the patients for 3 days. ${ }^{31}$ If the patient had no reaction during or following treatment, the diagnosis of allergy to BLs was ruled out.

As described by Park and others, ${ }^{25,32,33}$ patients with positive ST were not challenged because of the high risk of inducing a reaction. Each patient was carefully monitored during the whole procedure, with facilities and trained staff for managing any possible undesired and unexpected reactions. Patients with an HSR strongly suggestive of being IgE-mediated, with negative ST and tolerance of DCT with the selected BL, were reevaluated at 4 to 6 weeks to exclude a booster response and/or resensitization. Following published guidelines, we did not test patients with suspected severe NIHSR due to BLs. ${ }^{14,34,35}$

\section{Statistical Analysis}

Descriptive statistical tests were applied to assess patient characteristics, with continuous values expressed as means (standard deviation, SD) or medians (interquartile $25-75$, $\mathrm{IQ}_{25-75}$ ). Differences between groups were evaluated using the Student's $t$-test for unpaired data when the sample followed a normal distribution, or using the MannWhitney $U$-test for non-parametric variables. For paired samples that did not follow a normal distribution, the Wilcoxon test was used. The chi-squared test or Fisher's exact test was applied to analyze categorical variables. The odds of being truly allergic was assessed by means of multiple logistic regression analysis. 
Little's MCAR test was used to determine if the missing data followed the same distribution as available data. Multiple imputations by chained equations were performed, and a multivariable logistic regression model fitted.

The SPSS program version 25 (IBM, USA) and the $\mathrm{R}$ package version 3.5.1 were used to perform statistical analyses; $p$ values $<0.05$ were considered significant.

\section{Results}

Of the 1362 patients who reported allergy to BLs, $62 \%$ agreed to participate, and the allergological study was completed in 565 (286 in group A and 279 in group B (Figure 1). Patients' characteristics are shown in Table 1 . There were more women than men in both groups $(p=0.03)$. The time between occurrence of the HSR and performance of the study was shorter in group A than in group B (median 5 years versus 30 years; $p<0.01$ ).

An allergist had previously evaluated and confirmed BL allergy in 22 patients in group A and 7 in the group B. Of these, 16 and 4 cases, respectively, were IHSR and the others presented NIHSR.

A total of 175 patients $(93 \%$ of whom belonged to group B) including some of those who were previously confirmed as allergic were treated with a BL at some time before being studied by us, despite being labeled as allergic.

Clinical data (Table 1) showed that $88.8 \%$ of patients from group A and $54.5 \%$ from group B provided detailed information about the symptoms that occurred in the initial HSR, allowing us to classify the reactions as IHSR and NIHSR. Precise information of the reactions was not available in $11.2 \%$ of patients in group $\mathrm{A}$ and $45.6 \%$ in group B $(p<0.01)$.

According to the clinical history, skin was the most commonly involved organ (Figure 2). Urticaria was the most frequent clinical manifestation in group A (33.2\%) and maculopapular exanthema (MPE) in group B (20.8\%). Anaphylaxis was reported in $15.4 \%$ of patients in group A and $3.2 \%$ in group B $(p<0.01)$.

The BLs involved in the HSR are shown in Table 2. There was a clear difference in the culprit drug between groups: amoxicillin, amoxicillin-clavulanic acid and ampicillin were more frequent in group A $(54.5 \% ; p<0.01)$, while benzylpenicillin was more frequent in group B $(59.8 \% ; p<0.01)$.

ST were positive in $17.8 \%$ of the 285 group A patients, and in $2.9 \%$ of the 267 group B patients $(p<0.01)$. The

\section{Group A}

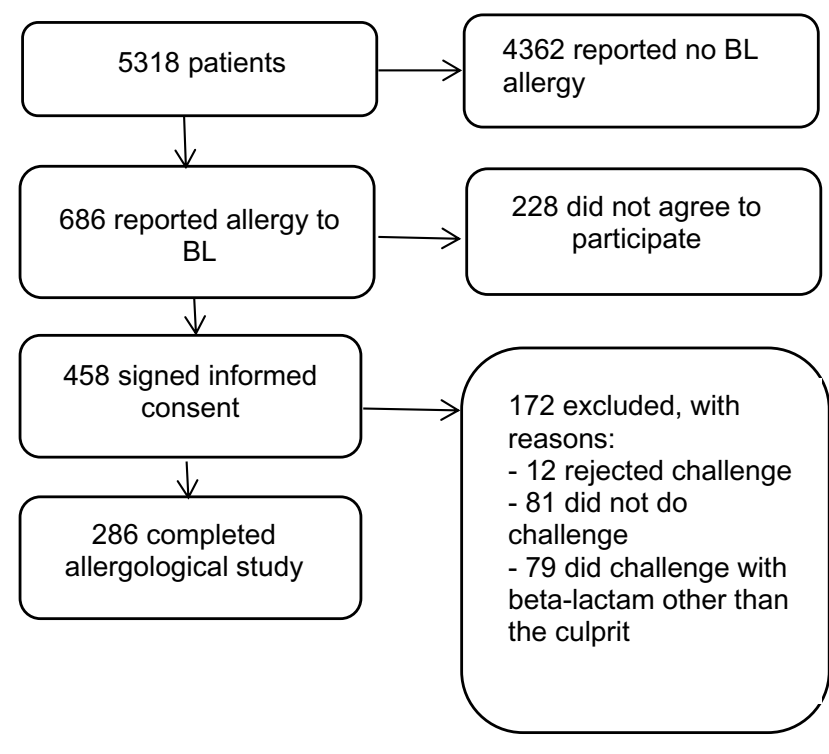

\section{Group B}

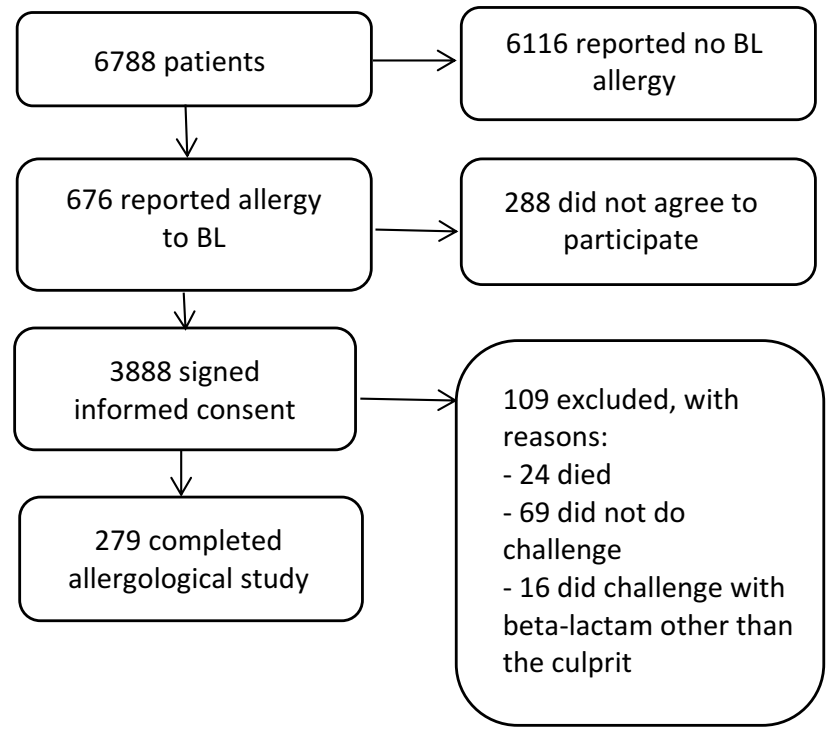

Figure I Participant flow chart.

determinants involved in the respective groups were mainly major and/or minor benzylpenicillin determinants $(39.2 \%$ versus $87.5 \% ; p<0.01)$ and amoxicillin and/or ampicillin (39.2\% versus $12.5 \%$; $p<0.01)$. A small proportion $(7.8 \%)$ of cases in group A presented positive results on ST to cephalosporins, compared to none in group B. Only $2 \%$ of the group A participants were ST positive to clavulanic acid (Tables 3 and 4).

Regarding the clinical presentation in patients with positive ST, anaphylaxis was the most frequent in both groups ( $48 \%$ and $62.5 \%$, respectively). The logistic 
Table I Participants' Characteristics

\begin{tabular}{|c|c|c|c|}
\hline Variables & $\begin{array}{l}\text { Group A } \\
(n=286)\end{array}$ & $\begin{array}{l}\text { Group B } \\
(n=279)\end{array}$ & $p$ value \\
\hline Age, years, median $\left(\mathrm{IQ}_{25-75}\right)$ & $67(10)$ & $85(6)$ & \\
\hline Women, n (\%) & $189(66.1)$ & $207(74.2)$ & 0.03 \\
\hline \multicolumn{4}{|l|}{ Place of origin, $n(\%)$} \\
\hline Spain & 283 (98.9) & $263(94.3)$ & 0.02 \\
\hline Other countries $^{\mathrm{a}}$ & $3(1)$ & $16(5.7)$ & \\
\hline Atopy, n (\%) & $107(37.4)$ & $48(17.2)$ & $<0.01$ \\
\hline Allergy to other drugs, n (\%) & $69(24.1)$ & $59(21.1)$ & 0.35 \\
\hline $\begin{array}{l}\text { Years since the initial HSR, } \\
\text { median }\left(\mathrm{IQ}_{25-75}\right)\end{array}$ & $5(34.5)$ & $30(30)$ & $<0.01$ \\
\hline \multicolumn{4}{|l|}{ Timing of reaction, $\mathrm{n}(\%)$} \\
\hline Immediate & $149(52.1)$ & $101(36.2)$ & 0.12 \\
\hline Non-immediate & $105(36.7)$ & $51(18.3)$ & \\
\hline Do not remember & $32(11.2)$ & $127(45.6)$ & $<0.01$ \\
\hline \multicolumn{4}{|l|}{ Skin tests, n (\%) } \\
\hline Positive & $51(17.8)$ & $8(2.9)$ & $<0.01$ \\
\hline Negative & $234(81.8)$ & $255(91.4)$ & \\
\hline Inhibited/not done & I (0.3) & $16(5.7)$ & \\
\hline Allergic $^{\mathrm{b}}, \mathrm{n}(\%)$ & $77(26.9)$ & $15(5.4)$ & $<0.01$ \\
\hline
\end{tabular}

Notes: ${ }^{a}$ Other European and South American countries. ${ }^{b}$ Allergic is defined by patients with positive skin tests or drug challenge tests.

Abbreviations: $\mathrm{IQ}_{25-75}$, interquartile $25-75$; HSR, hypersensitivity reaction.

regression analysis showed that a history of anaphylaxis is a predictor of positive results in ST $(p<0.01)$.

DCT was done in 235 patients of group A $(99.6 \%$ by oral route) and in 270 patients of group B $(89.4 \%$ by oral route and $10.4 \%$ by intravenous route). It was well

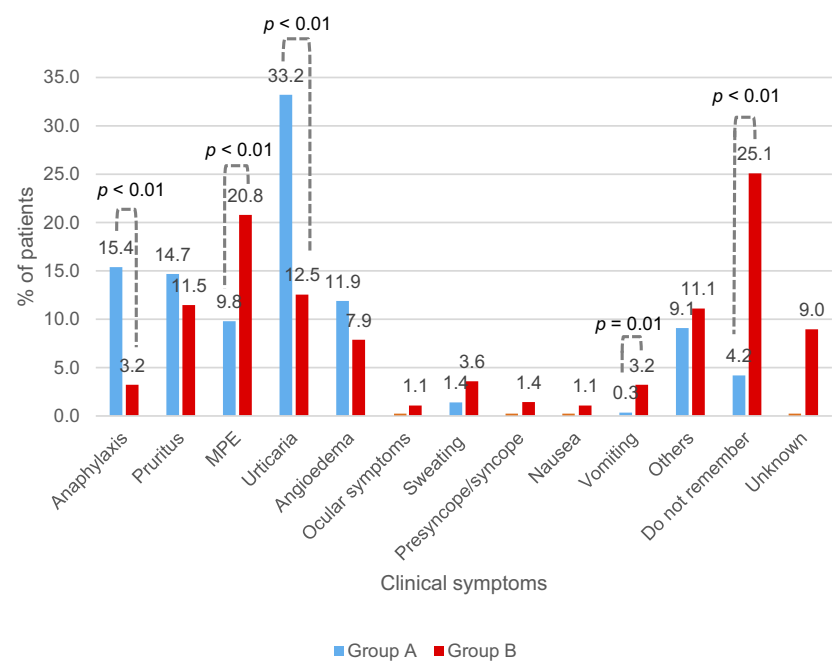

Figure 2 Clinical manifestations of the initial hypersensitivity reactions. The graph shows the symptoms of the HSR based on which the diagnosis of BL allergy was established. Being the most frequent cutaneous symptoms in both groups, while anaphylaxis was more frequent in group $A$. On the other hand, the majority of patients who did not remember the symptoms were those of group $B$.
Table 2 Beta-Lactams Involved in the Initial HSR by Age Group

\begin{tabular}{|l|l|l|l|}
\hline Culprit Drug & $\begin{array}{l}\text { Group A } \\
\text { N = 286 } \\
\text { n (\%) }\end{array}$ & $\begin{array}{l}\text { Group B } \\
\text { N = 279 } \\
\text { n (\%) }\end{array}$ & p value \\
\hline Benzylpenicillin & $96(33.6)$ & $167(59.8)$ & $<0.01$ \\
Amoxicillin & $82(28.7)$ & $20(7.2)$ & $<0.01$ \\
Amoxicillin/clavulanic acid & $73(25.5)$ & $18(6.5)$ & \\
Ampicillin & $\mathrm{I}(0.3)$ & 0 & \\
Cloxacillin & $\mathrm{I}(0.3)$ & $3(1.1)$ & 0.30 \\
Ceftriaxone & $20(7)$ & $4(1.4)$ & 0.01 \\
Cefazoline & $2(0.7)$ & 0 & 0.16 \\
Cefuroxime & $2(0.7)$ & 0 & 0.16 \\
Meropenem & 0 & $4(1.4)$ & 0.04 \\
Imipenem & 0 & $2(0.7)$ & 0.15 \\
Piperacillin/tazobactam & $\mathrm{I}(0.3)$ & $\mathrm{I}(0.4)$ & 0.98 \\
Does not remember & $8(2.8)$ & $60(21.5)$ & $<0.01$ \\
\hline
\end{tabular}

tolerated in $89.4 \%$ and $97.8 \%$, respectively $(p<0.01)$. The drugs used in the challenges are shown in Table 5. Reactions during DCT were more frequent in group A $(10.6 \%$ versus $2.2 \%$, respectively, $p=0.007)$. We detected $\operatorname{sigE}$ in 6 of the 29 patients ( 3 in each group) who had previously been diagnosed as allergic but had negative skin tests and good tolerance in DCT.

Retest was done in 128 patients (84 in group A and 44 in group B) and only two patients (1.6\%) became positive. One was in a 63-year-old man with an episode of immediate angioedema from 40 years earlier, who had good tolerance in the DCT to amoxicillin (culprit drug) and became positive to this antibiotic after the challenge. The second case was an 85-year-old woman with a history of dizziness after being treated with benzylpenicillin from 40 years earlier, who developed an immediate urticaria during a DCT with penicillin $\mathrm{V}$ and became positive to penicillin on ST. The reaction was controlled with intravenous antihistamines.

Symptoms compatible with NIHSR were reported in 156 patients: $41.3 \%$ of group A and $33.6 \%$ of group B. MPE was the most frequent clinical entity reported in both groups ( $26.7 \%$ and $29.4 \%$, respectively), followed by non-immediate urticaria $(38.1 \%$ and $31.4 \%)$ and nonimmediate angioedema ( $18.1 \%$ and $5.9 \%)$. ST were negative on delayed reading in $94.9 \%$ of these cases. DCT was performed in $99.3 \%$ of patients, observing a positive nonimmediate response in 16 patients in group A (8 MPE, 7 non-immediate urticaria, and 1 angioedema) and 4 patients in group B (3 MPE and 1 erythroderma) $(p=0.007)$. In addition, one patient in group A developed an urticaria 50 


\begin{tabular}{|c|c|c|c|c|c|c|c|c|c|c|c|c|c|c|c|c|c|c|c|}
\hline $\begin{array}{l}\frac{y}{\underline{z}} \\
\bar{z} \\
\check{x} \\
\underline{u} \\
\underline{\Delta}\end{array}$ & & $\stackrel{+}{+}$ & & & 111 & & & & & & & & & $\stackrel{9}{+}$ & $\mp$ & & & $\stackrel{0}{+}$ & \\
\hline 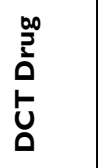 & O & نे & $\mathrm{O}$ & Q & z & ○ & o & O & o & $\stackrel{\circ}{z}$ & o & o & $\stackrel{\circ}{z}$ & $\underset{<}{x}$ & 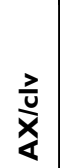 & O & $\mathrm{O}$ & $\underset{<}{x}$ & O \\
\hline 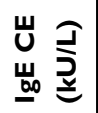 & $\overline{0}$ & 0 & $\stackrel{n}{0}$ & $\overline{\dot{v}}$ & 0 & 0 & $\mathrm{Q}$ & $\overline{\mathrm{v}}$ & $\overline{\dot{v}}$ & $\overline{\mathrm{v}}$ & $\bar{i}$ & $\overline{\mathrm{v}}$ & $\overline{\mathrm{v}}$ & $\overline{\dot{v}}$ & $\overline{\dot{v}}$ & $\overline{0}$ & $\overline{\mathrm{v}}$ & $\overline{\dot{v}}$ & 0 \\
\hline 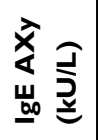 & 号 & 0 & ơ & $\overline{\mathrm{v}}$ & 0 & 0 & $\mathrm{Q}$ & $\overline{\mathrm{i}}$ & $\overline{\dot{v}}$ & $\overline{\dot{v}}$ & $\overline{\dot{v}}$ & $\overline{\dot{v}}$ & $\overline{\mathrm{v}}$ & $\overline{\dot{v}}$ & $\overline{\mathrm{v}}$ & $\overline{\dot{v}}$ & $\overline{\dot{v}}$ & $\overline{\mathrm{v}}$ & 0 \\
\hline 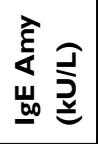 & 0 & 0 & 0 & $\overline{\mathrm{v}}$ & 0 & సี & $\mathrm{Q}$ & $\overline{\mathrm{v}}$ & $\overline{\dot{v}}$ & $\overline{\dot{v}}$ & ờ & $\overline{\dot{v}}$ & $\overline{\dot{v}}$ & $\overline{\dot{v}}$ & $\overline{\dot{0}}$ & $\overline{\dot{v}}$ & $\overline{\mathrm{v}}$ & $\overline{\dot{v}}$ & $\frac{\pi}{0}$ \\
\hline 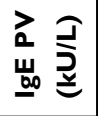 & 范 & 0 & o̊. & $\overline{\dot{v}}$ & 0 & 0 & $\mathrm{Q}$ & $\overline{\mathrm{v}}$ & $\overline{\dot{v}}$ & $\overline{\mathrm{v}}$ & $\overline{\mathrm{v}}$ & $\bar{i}$ & $\overline{\dot{v}}$ & $\overline{\dot{v}}$ & $\overline{\dot{0}}$ & $\overline{\dot{v}}$ & $\overline{\dot{v}}$ & $\overline{\dot{v}}$ & 0 \\
\hline 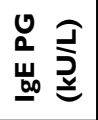 & mo & 0 & $\overline{0}$ & $\overline{\dot{v}}$ & 0 & $\stackrel{m}{0}$ & $\mathrm{Q}$ & $\overline{\mathrm{v}}$ & $\overline{\dot{v}}$ & $\overline{\dot{v}}$ & $\overline{\mathrm{v}}$ & $\overline{\dot{v}}$ & $\overline{\dot{v}}$ & $\overline{\dot{v}}$ & $\overline{\dot{v}}$ & $\overline{\vec{v}}$ & $\overline{\mathrm{v}}$ & $\overline{\dot{v}}$ & 0 \\
\hline 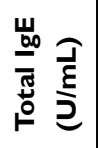 & $\begin{array}{l}0 \\
\dot{0} \\
\dot{0}\end{array}$ & $\stackrel{\infty}{\infty}$ & $\stackrel{\mathfrak{N}}{\mathfrak{N}}$ & $N$ & $\overline{\bar{\infty}}$ & $\lambda$ & $\mathrm{Q}$ & $\stackrel{\circ}{\varrho}$ & $\stackrel{\infty}{\infty}$ & $\stackrel{\kappa}{\underline{n}}$ & $\stackrel{\infty}{\wedge}$ & $\infty$ & n & $\stackrel{\sim}{\sim}$ & $\theta$ & in & $\stackrel{\stackrel{a}{m}}{n}$ & $\underline{n}$ & $\hat{\lambda}$ \\
\hline 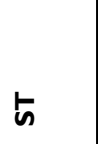 & $\begin{array}{l}x \\
\overleftarrow{4} \\
+\end{array}$ & 1 & $\begin{array}{ll}x & \Sigma \\
+ & 4 \\
+\end{array}$ & $\begin{array}{r}\frac{2}{\bar{y}} \\
\times \\
+\quad+4\end{array}$ & $\begin{array}{l}\geq \\
+\end{array}$ & $\begin{array}{l}z \\
+ \\
+\end{array}$ & 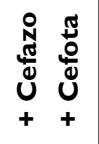 & $\begin{array}{l}x \\
< \\
+\end{array}$ & $\begin{array}{l}z \\
\mathbf{z} \\
+\end{array}$ & $\begin{array}{l}x \\
\times \\
+\end{array}$ & $\begin{array}{l}z \\
+ \\
+\end{array}$ & $\begin{array}{l}x \\
+ \\
+\end{array}$ & $\begin{array}{l}z \\
\mathbf{+} \\
+\end{array}$ & । & , & $\begin{array}{l}z \\
a \\
+\end{array}$ & $\begin{array}{l}z \\
z \\
0 \\
+\end{array}$ & । & $\begin{array}{l}x \\
+ \\
+\end{array}$ \\
\hline 胥 & $\frac{z}{2}$ & 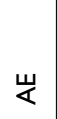 & $\frac{\pi}{\tilde{x}}$ & 5 & $\frac{2}{2}$ & $\frac{o}{z}$ & $\frac{\omega}{\underline{\Sigma}}$ & $\stackrel{\tilde{s}}{\frac{\pi}{4}}$ & 5 & 5 & $\stackrel{\pi}{\leftarrow}$ & \pm & 론 & 5 & 5 & $\frac{\widetilde{E}}{<}$ & $\underset{\leftarrow}{\tilde{E}}$ & 5 & $\frac{\pi}{\tilde{K}}$ \\
\hline 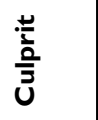 & $\frac{2}{\bar{x}}$ & 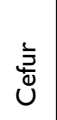 & $\underset{x}{x}$ & $\frac{2}{\bar{x}}$ & $\frac{2}{\bar{x}}$ & $z$ & 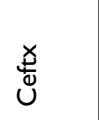 & $\stackrel{x}{<}$ & za & $\underset{4}{x}$ & $\underset{<}{x}$ & $\frac{x}{<}$ & $z$ & 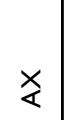 & $\frac{z}{\bar{x}}$ & $\frac{z}{\bar{y}}$ & $\frac{z}{\bar{x}}$ & $\frac{z}{\bar{x}}$ & $\underset{<}{x}$ \\
\hline 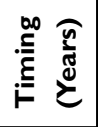 & $n$ & $\stackrel{\sim}{\sim}$ & $\stackrel{n}{o}$ & $\stackrel{\infty}{0}$ & $\stackrel{\infty}{\circ}$ & 오 & $\sigma$ & $\frac{0}{0}$ & $\stackrel{\infty}{0}$ & $\underline{n}$ & $\stackrel{\infty}{\circ}$ & $\stackrel{\infty}{\circ}$ & ㅇ & $\stackrel{\infty}{0}$ & $\begin{array}{l}\infty \\
0 \\
0\end{array}$ & $\begin{array}{l}\infty \\
0 \\
0\end{array}$ & ?口 & $\stackrel{\stackrel{\sim}{0}}{\circ}$ & i \\
\hline$\stackrel{8}{\&}$ & $\overline{6}$ & $\hat{0}$ & $\overline{6}$ & $\stackrel{\infty}{\wedge}$ & $\stackrel{n}{\wedge}$ & $\stackrel{N}{N}$ & $\pi$ & $\pi$ & $m$ & a & 8 & 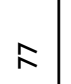 & $\tilde{\beta}$ & $\stackrel{\infty}{\infty}$ & $\infty$ & $\div$ & ธิ & $\hat{\sigma}$ & $\bar{\sigma}$ \\
\hline \# & - & $N$ & $m$ & $\sigma$ & in & 0 & n & $\infty$ & $a$ & 음 & $=$ & $\simeq$ & $\underline{m}$ & $\Xi$ & $\underline{n}$ & - & 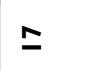 & $\underline{\infty}$ & $\underline{a}$ \\
\hline
\end{tabular}




\begin{tabular}{|c|c|c|c|c|c|c|c|c|c|c|c|c|c|c|c|c|c|c|c|}
\hline$\stackrel{+}{+}$ & Q & & & & $\stackrel{0}{+}$ & & & & & $\stackrel{+}{+}$ & $\mp$ & & & $\stackrel{q}{+}$ & & & $\stackrel{+}{+}$ & 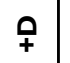 & $\stackrel{9}{+}$ \\
\hline$\underset{<}{x}$ & $z$ & $\stackrel{0}{z}$ & ₹ & ○ & $\underset{4}{x}$ & $\mathrm{O}$ & $\mathrm{O}$ & Q & $\mathrm{O}$ & $\stackrel{x}{<}$ & $\mathbf{z}$ & $\stackrel{\circ}{z}$ & o & $\underset{4}{x}$ & Oे & $\stackrel{\circ}{z}$ & $\underset{<}{x}$ & $\frac{z}{\bar{x}}$ & $\underset{<}{\times}$ \\
\hline 0 & $\overline{0}$ & $\bar{\circ}$ & 0 & 0 & $\bar{i}$ & $\overline{\dot{v}}$ & $\overline{\dot{v}}$ & 0 & 0 & 0 & $\mathrm{Q}$ & 0 & 0 & 0 & 0 & $\mathrm{Q}$ & 0 & $\overline{\hat{v}}$ & 0 \\
\hline 0 & $\bar{o}$ & $\stackrel{\text { no }}{0}$ & 0 & 0 & $\bar{i}$ & $\overline{\dot{v}}$ & $\overline{\mathrm{v}}$ & 0 & 0 & 0 & २ & 0 & ָָ & 0 & 0 & $\stackrel{m}{\underline{m}}$ & 0 & $\overline{\dot{v}}$ & 0 \\
\hline 0 & $\stackrel{m}{0}$ & 0 & 0 & 0 & $\overline{\mathrm{v}}$ & $\overline{\mathrm{v}}$ & $\overline{\mathrm{v}}$ & 0 & 0 & 0 & $\mathrm{Q}$ & 0 & 0 & 0 & 0 & $\frac{n}{\dot{r}}$ & 0 & $\overline{\dot{v}}$ & 0 \\
\hline 0 & $\bar{o}$ & ô. & 0 & 0 & $\overline{\dot{v}}$ & $\overline{\mathrm{v}}$ & $\overline{\dot{v}}$ & 0 & 0 & 0 & $\mathrm{Q}$ & 0 & 0 & 0 & 0 & 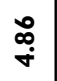 & 0 & $\overline{\dot{v}}$ & 0 \\
\hline 0 & $\begin{array}{l}0 \\
0 \\
0\end{array}$ & o. & 0 & 0 & $\overline{\mathrm{v}}$ & $\overline{\mathrm{v}}$ & $\overline{\dot{v}}$ & 0 & 0 & 0 & $\mathrm{Q}$ & 0 & 훙 & 0 & 0 & 惫 & 0 & $\overline{\dot{v}}$ & 0 \\
\hline$\grave{\sim}$ & $\underline{\Xi}$ & 想 & $\grave{N}$ & ભ్ల & f & $\underline{\underline{n}}$ & ㅇ & q & $\underline{q}$ & $\stackrel{t}{m}$ & $\mathrm{Q}$ & $\simeq$ & $\underset{\infty}{\circ}$ & นก & $\mathcal{F}$ & $\underline{\infty}$ & $n$ & $\stackrel{\infty}{\sim}$ & $\stackrel{\sim}{N}$ \\
\hline 1 & 1 & $\begin{array}{l}x \\
+ \\
+\end{array}$ & $\begin{array}{l}\frac{1}{a} z \\
+\quad+ \\
+\end{array}$ & $\begin{array}{l}z \\
+ \\
+\end{array}$ & 1 & $\begin{array}{l}z \\
a \\
+\end{array}$ & $\begin{array}{l}z \\
a \\
+\end{array}$ & $\begin{array}{l}x \\
< \\
+\end{array}$ & $\begin{array}{l}z \\
\mathbf{a} \\
+\end{array}$ & 1 & 1 & $\begin{array}{l}z \times< \\
z a \\
+\end{array}$ & $\begin{array}{lll}z & x & \frac{z}{\bar{v}} \\
a & \sum & \alpha \\
+ & + & +\end{array}$ & 1 & $\begin{array}{ll}\frac{a}{a} & z \\
a & \\
+ & +\end{array}$ & $\begin{array}{l}\frac{a}{a} \\
+\end{array}$ & , & 1 & 1 \\
\hline$\frac{u}{\frac{\omega}{\Sigma}}$ & 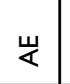 & 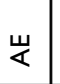 & $\stackrel{\widetilde{5}}{\frac{5}{4}}$ & 5 & 5 & $\stackrel{\widetilde{c}}{\frac{\pi}{4}}$ & 节 & $\stackrel{\widetilde{5}}{<}$ & $\stackrel{\pi}{\leftarrow}$ & $\frac{\alpha}{z}$ & 5 & 5 & $\underline{2}$ & 芒 & 䒓 & 5 & $\frac{\omega}{\frac{\omega}{\Sigma}}$ & $\frac{u}{\Sigma}$ & $\frac{\omega}{\Sigma}$ \\
\hline$\underset{\frac{2}{x}}{\frac{2}{x}}$ & $\underset{<}{x}$ & $\underset{<}{x}$ & $z$ & $\underset{<}{x}$ & $\frac{\frac{2}{4}}{\frac{x}{4}}$ & $\frac{2}{4}$ & $\frac{\geq}{\grave{x}}$ & $\underset{<}{x}$ & $\underset{<}{x}$ & ج & $\frac{o}{Z}$ & $\frac{\alpha}{z}$ & $\frac{2}{\mathrm{x}}$ & za & $\frac{2}{4}$ & $\frac{2}{\bar{x}}$ & $\underset{<}{x}$ & $\frac{z}{\bar{x}}$ & $\underset{<}{x}$ \\
\hline$\stackrel{\infty}{\circ}$ & $\stackrel{\infty}{\circ}$ & $\stackrel{n}{o}$ & ஓ & $\stackrel{\infty}{\circ}$ & $\stackrel{\infty}{0}$ & $\stackrel{\infty}{\circ}$ & นุ & $\stackrel{\infty}{\circ}$ & $\stackrel{2}{\sim}$ & 8 & ণ & g & $\stackrel{m}{0}$ & o & $\stackrel{\infty}{0}$ & 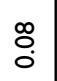 & $\stackrel{\infty}{\circ}$ & $N$ & $\stackrel{\infty}{0}$ \\
\hline$R$ & 8 & 8 & $\stackrel{n}{n}$ & N & $\stackrel{\infty}{\infty}$ & $\Xi$ & $\Sigma$ & 8 & tे & $\hat{\sigma}$ & $\hat{s}$ & 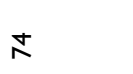 & $\overline{0}$ & $\overline{0}$ & นุ & $a$ & $\pi$ & R & $\tilde{3}$ \\
\hline 요 & $\bar{N}$ & $\tilde{N}$ & $\tilde{n}$ & d & $\stackrel{2}{\sim}$ & సి & $\tilde{N}$ & $\stackrel{\infty}{\sim}$ & ని & 品 & $\bar{m}$ & $\tilde{m}$ & $m$ & Jे & $\stackrel{n}{m}$ & $\stackrel{m}{m}$ & $\hat{m}$ & $\stackrel{\infty}{m}$ & ले \\
\hline
\end{tabular}




\begin{tabular}{|c|c|c|c|c|c|c|c|c|c|c|c|c|c|c|c|c|c|c|c|c|c|}
\hline 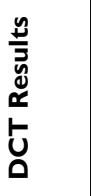 & & & & $\begin{array}{l}x \\
\times \\
+ \\
+ \\
+\end{array}$ & & c & & & & & & $\mp$ & & & & & & & $\stackrel{9}{+}$ & & $\mp$ \\
\hline 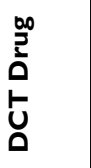 & Oे & Oे & Q & $\underset{x}{x}$ & $\frac{\mathrm{O}}{\mathrm{z}}$ & $\frac{1}{2}$ & & & & Oे & Oे & $\underset{x}{x}$ & O & Q & Oे & Oे & Q & O & $\begin{array}{l}x \\
<\end{array}$ & O & $\underset{x}{x}$ \\
\hline 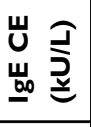 & 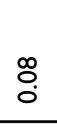 & $\overline{\dot{v}}$ & $\overline{\dot{v}}$ & ర్ & $\frac{\infty}{0}$ & $c$ & & & & 0 & 乙 & $\bar{\circ}$ & స్తి & 0 & 0 & 0 & $\overline{\mathrm{v}}$ & $\mathrm{Q}$ & 0 & $\overline{\mathrm{v}}$ & $\overline{\mathrm{v}}$ \\
\hline 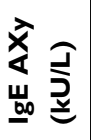 & $\overline{0}$ & $\overline{\mathrm{v}}$ & $\overline{\mathrm{i}}$ & ర్ & ¿’ & 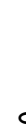 & & & & 0 & $\mathrm{Q}$ & $\bar{\circ}$ & $\stackrel{\infty}{i}$ & 0 & 0 & $\stackrel{\text { L }}{\circ}$ & $\overline{\dot{v}}$ & $\overline{-}$ & 0 & $\overline{\mathrm{v}}$ & $\overline{\mathrm{v}}$ \\
\hline 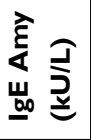 & 0 & $\overline{\dot{v}}$ & $\overline{\mathrm{O}}$ & 0 & $\stackrel{\substack{\infty \\
0 \\
0}}{0}$ & 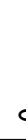 & & & & 0 & $\mathrm{Q}$ & 苍 & $\frac{a}{m}$ & 0 & 0 & ஜ̋ & $\overline{\mathrm{v}}$ & $\stackrel{\text { ஸे }}{\text { No }}$ & 0 & $\overline{\mathrm{v}}$ & $\bar{i}$ \\
\hline 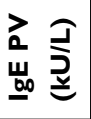 & 웅 & $\overline{\mathrm{v}}$ & $\overline{\dot{v}}$ & $\ddot{0}$ & $\stackrel{\text { an }}{0}$ & 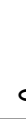 & & & & 0 & $\mathrm{Q}$ & o̊ & $\underset{\dot{\sigma}}{\mathfrak{j}}$ & 0 & 0 & $\bar{\circ}$ & $\overline{\mathrm{v}}$ & $\stackrel{\infty}{\stackrel{\infty}{-}}$ & 0 & $\overline{\mathrm{v}}$ & $\bar{i}$ \\
\hline 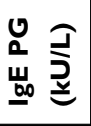 & 웅 & $\overline{\dot{v}}$ & $\overline{\mathrm{v}}$ & 0 & รั) & $c$ & & & & 0 & 之 & $\stackrel{\infty}{0}$ & $\begin{array}{l}\varpi \\
\dot{\Phi} \\
\dot{\varphi}\end{array}$ & 0 & 0 & 0 & $\overline{\mathrm{v}}$ & $\stackrel{\infty}{0}$ & 0 & $\overline{\mathrm{v}}$ & $\overline{\mathrm{v}}$ \\
\hline 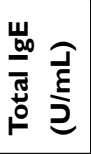 & 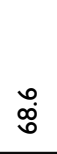 & 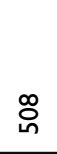 & $\tilde{0}$ & $\stackrel{\text { ํㅜ }}{=}$ & స్ స్ & 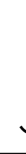 & & & & $\underline{\tilde{\delta}}$ & 吕 & $\bar{\swarrow}$ & $\widehat{\widehat{્}}$ & + & $N$ & 으 & $\stackrel{\infty}{\curvearrowleft}$ & సે & f & ه্ণ & 으 \\
\hline 占 & $\underset{+}{+}$ & $\begin{array}{l}\frac{2}{\bar{y}} \\
\frac{x}{4} \\
+\end{array}$ & $\underset{+}{x}$ & I & อัँ & & & & & $\begin{array}{l}x \\
+\end{array}$ & 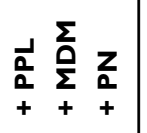 & 1 & $\frac{a}{a}$ & $\begin{array}{l}z \\
a \\
+\end{array}$ & $\begin{array}{l}z \\
a \\
+\end{array}$ & 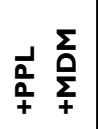 & $\begin{array}{l}x \\
+\end{array}$ & $\begin{array}{l}\frac{a}{a} \\
+\end{array}$ & । & $\begin{array}{l}\sum_{2} \\
\sum_{0} \\
+\end{array}$ & 1 \\
\hline Uू & $\frac{\omega}{\frac{\omega}{\Sigma}}$ & 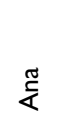 & 5 & 山्山 & $\frac{\pi}{4}$ & 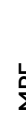 & & & & $\stackrel{\widetilde{2}}{\frac{\pi}{<}}$ & 荌 & \pm & 5 & 5 & $\frac{2}{2}$ & 5 & $\underset{\frac{\tilde{E}}{<}}{ }$ & 苛 & 5 & 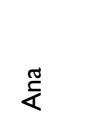 & 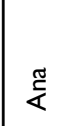 \\
\hline 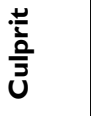 & $\sum$ & $\frac{2}{\frac{x}{x}}$ & $\frac{2}{\bar{x}}$ & $\underset{\alpha}{x}$ & 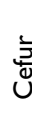 & 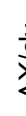 & & & & $\frac{z}{\vec{x}}$ & za & $\underset{<}{x}$ & $z$ & $\underset{<}{x}$ & $\underset{<}{x}$ & $\frac{z}{\bar{x}}$ & $\underset{<}{x}$ & $\frac{2}{\overrightarrow{0}}$ & $\underset{<}{x}$ & $\underset{<}{x}$ & 宏 \\
\hline 竞 & q & 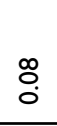 & $\stackrel{\infty}{\circ}$ & q & $\stackrel{\infty}{\circ}$ & $\hat{c}$ & & & & $\stackrel{\infty}{0}$ & in & $\frac{0}{0}$ & o & $\stackrel{\infty}{\circ}$ & $\stackrel{\llcorner}{0}$ & n & 0 & $\stackrel{2}{0}$ & i & $\stackrel{m}{o}$ & $\begin{array}{l}\text { त् } \\
\text { ஸे }\end{array}$ \\
\hline 橤 & $\stackrel{n}{\kappa}$ & 6 & $\overline{6}$ & กิ & 2 & $r$ & & & & ț & $\stackrel{m}{n}$ & $\overline{6}$ & $m$ & N & $R$ & $\hat{o}$ & N & $R$ & 8 & 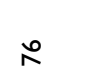 & 8 \\
\hline \# & \& & $\bar{\nabla}$ & $\mathcal{F}$ & $\tilde{\sigma}$ & 8 & 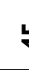 & & & & $\stackrel{\infty}{\sim}$ & $q$ & 오 & $\bar{n}$ & n & n & ir & in & in & in & $\stackrel{\infty}{n}$ & in \\
\hline
\end{tabular}




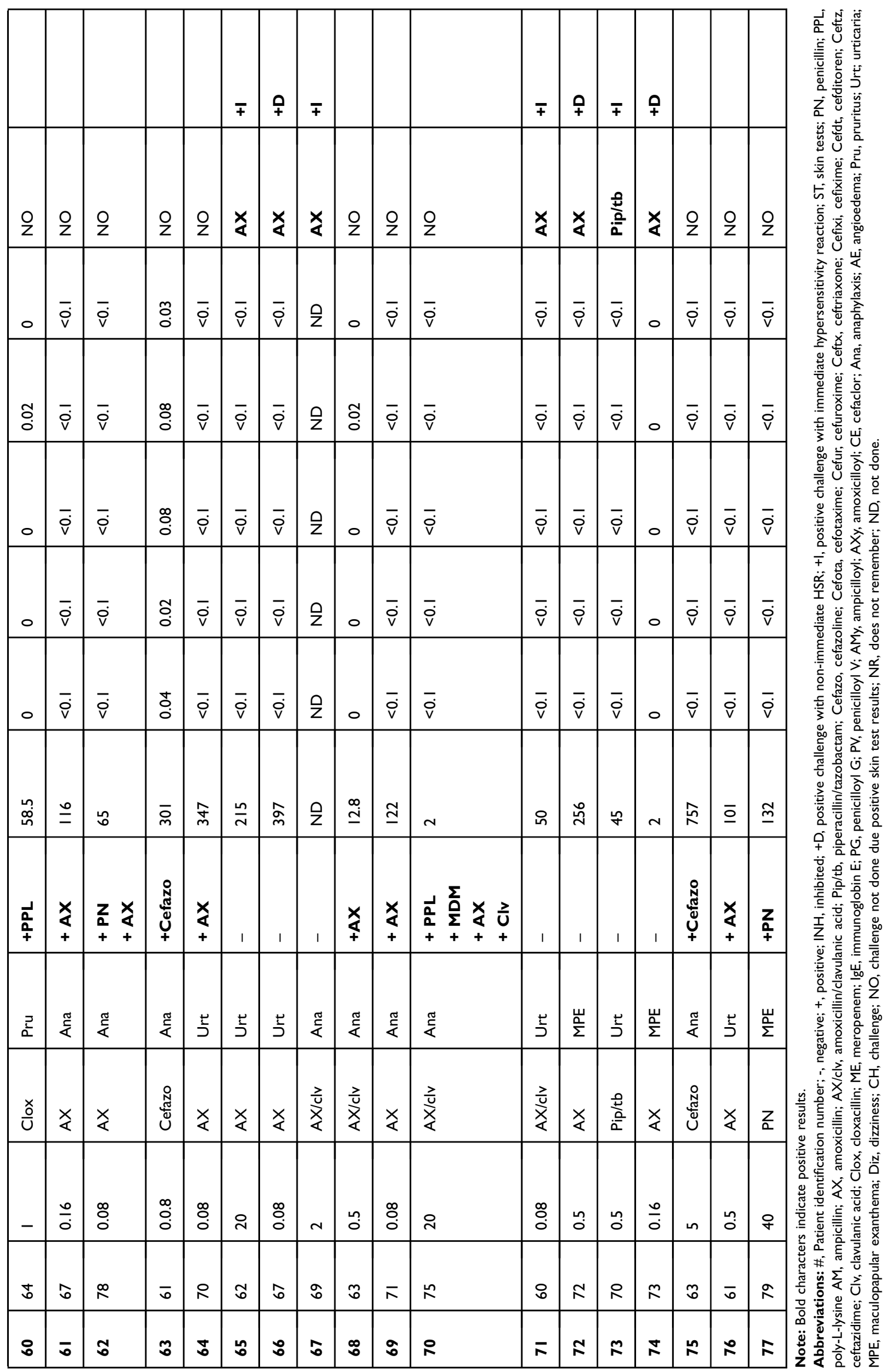




\begin{tabular}{|c|c|c|c|c|c|c|c|c|c|c|c|c|c|c|c|}
\hline 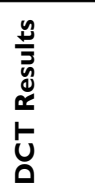 & $\stackrel{+}{+}$ & $\stackrel{+}{+}$ & & & & & 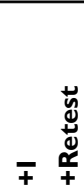 & & $\mp$ & $\stackrel{0}{+}$ & & & $\mp$ & & \\
\hline 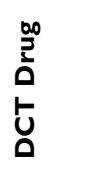 & U⿺辶ِ & $\stackrel{x}{<}$ & O & $\mathrm{o}$ & $\mathrm{O}$ & $\mathrm{O}$ & $\mathbf{z}$ & O & $z$ & $z_{a}$ & Oे & $\stackrel{0}{z}$ & $\frac{7}{\frac{x}{4}}$ & & \\
\hline 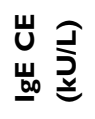 & $\begin{array}{l}0 \\
0 \\
0\end{array}$ & $\stackrel{m}{0}$ & ô. & $\overline{\overline{0}}$ & $\mathrm{Q}$ & $\stackrel{m}{0}$ & 0 & $\bar{v}$ & $\overline{\mathrm{v}}$ & $\overline{\dot{v}}$ & $\overline{\dot{v}}$ & $\overline{\mathrm{v}}$ & $\overline{\mathrm{v}}$ & & \\
\hline 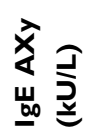 & $\stackrel{\infty}{0}$ & $\begin{array}{l}\text { t) } \\
0\end{array}$ & స్ర & $\frac{\infty}{0}$ & 乙 & òे & 0 & $\overline{\mathrm{v}}$ & $\bar{i}$ & $\overline{\hat{v}}$ & $\overline{\hat{v}}$ & $\overline{\mathrm{o}}$ & $\overline{\mathrm{o}}$ & & \\
\hline 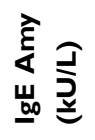 & $\stackrel{\text { 응 }}{\circ}$ & $\ddot{\circ}$ & ్ָ & $\stackrel{m}{\circ}$ & ₹ & 0 & $\frac{\infty}{0}$ & $\overline{\mathrm{v}}$ & $\overline{\mathrm{v}}$ & $\overline{\dot{v}}$ & $\overline{\dot{v}}$ & $\overline{\mathrm{v}}$ & $\overline{\mathrm{v}}$ & & \\
\hline 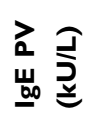 & $\begin{array}{l} \pm \\
0 \\
0\end{array}$ & $\overline{0}$ & กิ & $\frac{n}{0}$ & $\mathrm{z}$ & $\bar{ָ}$ & 0 & $\overline{\mathrm{v}}$ & $\overline{\mathrm{v}}$ & $\overline{\dot{v}}$ & $\overline{\dot{v}}$ & $\overline{\mathrm{v}}$ & $\overline{\mathrm{v}}$ & & \\
\hline 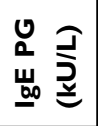 & $\bar{o}$ & 0 & ָ̊ & $\frac{n}{0}$ & ₹ & సָ & 0 & $\overline{\mathrm{v}}$ & $\overline{\mathrm{v}}$ & $\overline{\dot{v}}$ & $\overline{\dot{v}}$ & $\overline{\mathrm{v}}$ & $\overline{\dot{v}}$ & & \\
\hline 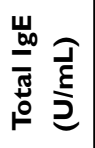 & $\frac{\dot{J}}{\vec{g}}$ & $\stackrel{m}{\infty}=$ & ڤ્' & 点 & $\mathrm{Q}$ & 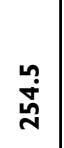 & $\sim$ & $\sigma$ & $a$ & $\stackrel{ \pm}{\sim}$ & $\hat{m}$ & 으 & $a$ & & \\
\hline 占 & 1 & 1 & $\begin{array}{lll}z & \times & \Sigma \\
a & < & 4 \\
+ & + & +\end{array}$ & $\begin{array}{l}\stackrel{x}{<} \\
+\end{array}$ & $\begin{array}{l}z \\
\mathbf{z} \\
+\end{array}$ & $\begin{array}{l}\frac{1}{2} \\
+ \\
+\end{array}$ & 1 & $\begin{array}{l}z \\
\text { a }\end{array}$ & । & I & $\sum_{+}^{\sum_{+}} z_{0}$ & $\begin{array}{l}z \\
a \\
+\end{array}$ & I & & \\
\hline 矛 & 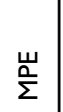 & 訔 & $\stackrel{\pi}{\frac{\pi}{\alpha}}$ & $\underset{\frac{\pi}{4}}{2}$ & $\underset{\frac{\pi}{\alpha}}{2}$ & $\stackrel{\pi}{\frac{\tilde{x}}{<}}$ & $\stackrel{N}{\Delta}$ & $\frac{2}{2}$ & 山्山 & $\frac{\omega}{\Sigma}$ & $\stackrel{N}{\Delta}$ & $\stackrel{\alpha}{z}$ & $\frac{\omega}{\Sigma}$ & & \\
\hline 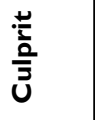 & 希 & $\underset{<}{\times}$ & $\underline{z}$ & $\stackrel{o}{Z}$ & 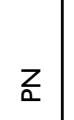 & za & $\underline{z}$ & z & $\underline{z}$ & $\frac{e}{a}$ & $z$ & $\frac{\alpha}{z}$ & $\frac{3}{\bar{x}}$ & & \\
\hline 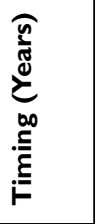 & $\stackrel{\text { ำ }}{0}$ & นุ & ஓి & 요 & 요 & 8 & 아 & p & 요 & $\frac{1}{0}$ & 요 & i & तु & & \\
\hline 品 & $\infty$ & $\bar{\infty}$ & 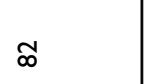 & $a$ & $\infty$ & $\bar{\infty}$ & $\stackrel{\llcorner}{\infty}$ & 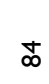 & న & œ & ळ & $\bar{\infty}$ & $\infty$ & & \\
\hline$\#$ & - & $N$ & $m$ & $\sigma$ & in & 0 & $n$ & $\infty$ & $a$ & - & $=$ & \pm & $\underline{-}$ & & \\
\hline
\end{tabular}


Table 5 Drug Challenges and Antibiotic Selected for Them

\begin{tabular}{|c|c|c|c|c|c|c|}
\hline \multirow[t]{2}{*}{ Culprit Drug } & \multicolumn{3}{|c|}{ Group A; N = 286} & \multicolumn{3}{|c|}{ Group B; N =279 } \\
\hline & $\mathbf{n}$ & Drug Challenge & Total & $\mathbf{n}$ & Drug Challenge & Total \\
\hline Penicillin & 96 & $\begin{array}{l}\text { Amoxicillin } \\
\text { Amoxicillin/clavulanic acid } \\
\mathrm{CH} \text { not done } \mathrm{a}^{\mathrm{a}}\end{array}$ & $\begin{array}{l}71 \\
18 \\
7\end{array}$ & 167 & $\begin{array}{l}\text { Penicillin } \\
\text { Amoxicillin } \\
\text { Amoxicillin/clavulanic acid } \\
\text { Ampicillin } \\
\mathrm{CH} \text { not done }{ }^{\mathrm{a}}\end{array}$ & $\begin{array}{l}2 \\
107 \\
49 \\
4 \\
5\end{array}$ \\
\hline Amoxicillin & 82 & $\begin{array}{l}\text { Penicillin } \\
\text { Amoxicillin } \\
\text { Amoxicillin/clavulanic acid } \\
\mathrm{CH} \text { not done }{ }^{\mathrm{a}}\end{array}$ & $\begin{array}{l}1 \\
52 \\
10 \\
19\end{array}$ & 20 & $\begin{array}{l}\text { Amoxicillin } \\
\text { Amoxicillin/clavulanic acid } \\
\text { Ampicillin } \\
\mathrm{CH} \text { not done }{ }^{\mathrm{a}}\end{array}$ & $\begin{array}{l}13 \\
3 \\
3 \\
1\end{array}$ \\
\hline Amoxicillin/ clavulanic acid & 73 & $\begin{array}{l}\text { Amoxicillin } \\
\text { Amoxicillin/clavulanic acid } \\
\mathrm{CH} \text { not done } \mathrm{a}^{\mathrm{a}}\end{array}$ & $\begin{array}{l}5 \\
50 \\
18\end{array}$ & 18 & $\begin{array}{l}\text { Amoxicillin/clavulanic acid } \\
\mathrm{CH} \text { not done }{ }^{\mathrm{b}}\end{array}$ & $\begin{array}{l}17 \\
1\end{array}$ \\
\hline Ampicillin & I & $\mathrm{CH}$ not done $\mathrm{e}^{\mathrm{a}}$ & I & & & \\
\hline Cloxacillin & I & $\mathrm{CH}$ not done $\mathrm{a}^{\mathrm{a}}$ & I & 3 & Cloxacillin & 3 \\
\hline Ceftriaxone & 20 & $\begin{array}{l}\text { Cefuroxime } \\
\mathrm{CH} \text { not done }{ }^{\mathrm{a}}\end{array}$ & $\begin{array}{l}19 \\
1\end{array}$ & 4 & $\begin{array}{l}\text { Cefditoren } \\
\text { Cefuroxime }\end{array}$ & $\begin{array}{l}3 \\
1\end{array}$ \\
\hline Cefazoline & 2 & $\mathrm{CH}$ not done $\mathrm{e}^{\mathrm{a}}$ & 2 & & & \\
\hline Cefuroxime & 2 & $\begin{array}{l}\text { Cefuroxime } \\
\mathrm{CH} \text { not done }\end{array}$ & $\begin{array}{l}1 \\
1\end{array}$ & & & \\
\hline Meropenem & & & & 4 & Meropenem & 4 \\
\hline Imipenem & & & & 2 & Meropenem & 2 \\
\hline Piperacillin/tazobactam & I & Piperacillin/tazobactam & I & I & Penicillin & I \\
\hline Does not remember & 8 & $\begin{array}{l}\text { Penicillin } \\
\text { Amoxicillin } \\
\mathrm{CH} \text { not done }{ }^{\mathrm{a}}\end{array}$ & $\begin{array}{l}1 \\
6 \\
1\end{array}$ & 60 & $\begin{array}{l}\text { Penicillin } \\
\text { Amoxicillin } \\
\text { Ampicillin } \\
\mathrm{CH} \text { not done } \mathrm{e}^{\mathrm{a}}\end{array}$ & $\begin{array}{l}33 \\
22 \\
3 \\
2\end{array}$ \\
\hline
\end{tabular}

Notes: ${ }^{a} \mathrm{CH}$ not done due to positive skin test. ${ }^{\mathrm{b}} \mathrm{CH}$ not done due to anaphylaxis with slgE positive.

Abbreviation: $\mathrm{CH}$, challenge.

min after drug intake, which we considered an immediate HSR. All cases were controlled with corticoids and antihistamines at $12 \mathrm{hrs}$ to $24 \mathrm{hrs}$, and complete clearance was achieved within $72 \mathrm{hrs}$. Of note is that the previously confirmed cases in our study developed good tolerance.

Final diagnosis of allergy to $\mathrm{BL}$ was based on the results of skin testing and DCT, and was made in 77 (26.9\%) patients in group A and $15(5.4 \%)$ patients in group B $(p<0.01)$. Multivariable analysis showed that, after adjusting for sex, atopy, allergy to other drugs, and sIgE to amoxicilloyl, younger age is an independent risk factor for allergy to $\mathrm{BL}(p<0.01)$.

\section{Discussion}

Our aim was to confirm if patients' past BL allergy still remained and to establish the relationship between decreased immune response and the presence of allergy. Based on current knowledge regarding immunity in the very elderly ${ }^{20}$ our hypothesis was that age-related decline of both cellular and humoral immune response ${ }^{36-38}$ could result in tolerance to BLs in patients who had previous allergies with IgE- or T cell-mediated reactions. For this purpose, we compared two groups of patients, the first aged 60 to 79 years, and the second aged 80 or older. Participants had been labeled as allergic, so we evaluated 
their current status of allergy to BLs. The assessment included a DCT as the final proof of drug allergy or tolerance.

We found that a significant number of patients, mainly in the older group, had been treated with BLs-despite having a history of allergy to BL and in some cases with active alarm systems in medical records - without reporting any adverse reaction. These observations ${ }^{20}$ and the fact that 29 patients had been diagnosed by an Allergist, led us to undertake this study.

The profile of our cases reflects variations of the antibiotics use and allergological response according to age: we found patients, especially among the older group, who had reactions with penicillins and positive results to polyL-lysine, MDM, and/or penicillin, amoxicillin, ampicillin or amoxicillin/clavulanic acid. These correspond to common reactions to $\mathrm{BPO}$, the main determinant generated by benzylpenicillin. ${ }^{39,40}$ On the other hand, we observed many patients in the 60-79-year-old group with BLselective reactions to the side chain ${ }^{14,41,42}$ due to the more frequent prescription and use of amoxicillin, amoxicillin/clavulanic and ampicillin than BP. These patients reacted exclusively to amoxicillin, ampicillin or amoxicillin/clavulanic acid but were negative to both penicillin and its major and minor determinants. ${ }^{40,43}$ Positivity to ampicillin in our study was probably due to cross-reactivity with amoxicillin, as reported previously. ${ }^{44}$ There were also patients who reacted to cephalosporins, mainly in the younger group, who also had side chain-specific reactions, as previously reported. ${ }^{28,45,46}$

In participants' clinical histories, anaphylaxis was much more frequent in group A than in B, which is logical in light of the different reactions described above. In side chain-specific reactions, authors have observed that anaphylaxis is much more frequent than in classical benzylpenicillin cross-reactors, although this clinical entity occurs in both groups. ${ }^{47}$

A total of 286 patients aged 60 to 79 years and 279 aged 80 years or older completed the study, and $26.9 \%$ and $5.4 \%$, respectively, were confirmed as having a BL allergy $(p<0.01)$. Most participants showed good tolerance to penicillins and other BLs like cephalosporins (ceftriaxone, cefazoline, cefotaxime, cefuroxime). The natural loss of sensitization is one possible explanation for these findings, as this has been reported to occur more quickly in side chain-specific reactions like those to amoxicillin or cephalosporins..$^{28,42,43}$ In contrast, this phenomenon occurs more slowly for BPO-common reactions, also known to be cross-reactive within the penicillin group. ${ }^{10,48}$

The levels and function of different immunoglobulin isotypes have been studied in detail in the elderly. In murine models, Turner et al demonstrated that changes in the architecture of the lymph node and the spleen impaired the production of antibodies. ${ }^{49}$ Bourcy et al demonstrated the expansion of B-cell clones containing mostly IgM or IgA isotypes, which were unable to differentiate between functioning and non-functioning receptors. ${ }^{18}$

Sugahara et al have shown that in humans, the levels and binding capacity of IgA did not change with age, but its affinity decreased, apparently due to the loss of the function of the $\mathrm{T}$ lymphocytes in promoting antigenspecific IgA response via differentiation to $\mathrm{T}$ follicular helper cells. ${ }^{19}$ Regarding IgE however, an extensive literature search yielded no studies concerning the decline in this immunoglobulin response, particularly that related to IgE and drug HSR.

It is reasonable to think that, as with the other antibody isotypes, the decrease in the humoral response could entail a decrease in the affinity of IgE for the antigens, which would lead to unresponsiveness. This could explain why patients with IHSR do not develop reactions after reexposure to BLs. Although this is a strong hypothesis, new studies on IgE production, regulation and response in elderly people allergic to beta-lactams are necessary to clarify it. Regarding NIHSR, in our study, nine cases were previously confirmed by an allergist by DCT, four of these patients (group A) had positive results in DCT. Evidence suggests that $\mathrm{T}$ cell responses persist for a long time, with positive skin tests at the delayed intradermal reading. ${ }^{30,50}$ Thus, it is no surprise that we found patients with positive responses despite the time elapsed. However, the lower response in the older group may also be related to immunosenescence.

Regarding resensitization, we observed only $1.6 \%$ positivity after challenge with BLs. Considering that the rate of resensitization does not exceed that in healthy patients treated with BLs, retesting should not be routine as this is rather uncommon, and is recommended only in cases in which there is a high suspicion of IgE-mediated HSRs with negative ST and good tolerance verified in the DCT. ${ }^{2,6,51,52}$

One of the major problems in this population are bacterial infections, stemming from diminished humoral and cellular immunity. ${ }^{18,36,37,49}$ Therefore, patients labeled as allergic to $\mathrm{BL}$ are of particular concern and have attracted 
special attention because they may be deprived of treatments with these antibiotics, as Trubiano et al and others have pointed out. ${ }^{13,40,53-59}$ According to our results, older people could benefit from treatment with BLs if aging contributes to the loss of sensitivity, avoiding exposure to more toxic antibiotics like vancomycin and quinolones among others.

The main weakness of this study is that not all cases labelled as allergic in the past had a confirmed diagnosis. On the other hand, $45.6 \%$ of patients aged 80 years or older did not remember the event that led to the diagnosis of allergy (median 30 years earlier), so it was not possible to classify HSRs accurately. Therefore, allergological diagnosis cannot be established exclusively with the data collected in the clinical history as reported in previous studies. $^{54,60}$

The results of the multivariable analysis showed that the risk of allergy to BLs is lower in older patients. The logistic regression analysis showed that a history of anaphylaxis is a predictor of positive results in ST and should be taken into account when exposing patients to BLs again.

\section{Conclusion}

To our knowledge, this is the first allergological study conducted in elderly people. Allergy to BLs was confirmed in a low percentage of patients claiming to be allergic, being rare in the elderly over 80 years. It is known that a false labeling of allergies and spontaneous loss of sensitivity to BLs over time are facts related to this, but we describe for the first time that patients with a confirmed diagnosis in the past do not respond when they are re-exposed, this could be justified by the immunosenescence. However, considering this is an observational study, future experimental studies to elucidate what is happening in relation to the production and functioning of $\mathrm{IgE}$ during immunosenescence could help to generate more knowledge of beta-lactam allergy in the elderly.

\section{Acknowledgments}

The authors would like to thank Ms Meggan Harris and Dr Alexis Lara Rivero for the English edition of the manuscript.

\section{Disclosure}

Prof. Dr Javier Fernandez-Sanchez reports a grant from Carlos III Institute: RD16/06/032, during the conduct of the study. The authors report no other conflicts of interest in this work.

\section{References}

1. Ariza A, Mayorga C, Fernández TD, et al. Hypersensitivity reactions to ß-lactams: relevance of hapten-protein conjugates. J Investig Allergol Clin Immunol. 2015;25(1):12-25.

2. Solensky R. Allergy to beta-lactam antibiotics. J Allergy Clin Immunol. 2012;130(6):0-5. doi:10.1016/j.jaci.2012.08.021

3. Macy E, Poon K-Y T. Self-reported antibiotic allergy incidence and prevalence: age and sex effects. Am J Med. 2009;122(8):778.e1-778. e7. doi:10.1046/j.1365-2222.2003.01638.x

4. Lee CE, Zembower TR, Fotis MA, et al. The incidence of antimicrobial allergies in hospitalized patients: implications regarding prescribing patterns and emerging bacterial resistance. Arch Intern Med. 2000;160(18):2819-2822. doi:10.1001/archinte.160.18.2819

5. Macy E, Contreras R. Health care use and serious infection prevalence associated with penicillin "allergy" in hospitalized patients: a cohort study. J Allergy Clin Immunol. 2014;133(3):790-796. doi:10.1016/j.jaci.2013.09.021

6. Solensky R, Khan D. Drug allergy: an updated practice parameter. Ann Allergy Asthma Immunol. 2010;105(4):259-273.e78.

7. Sade K, Holtzer I, Levo Y, Kivity S. The economic burden of antibiotic treatment of penicillin-allergic patients in internal medicine wards of a general tertiary care hospital. Clin Exp Allergy. 2003;33 (4):501-506. doi:10.1046/j.1365-2222.2003.01638.x

8. Satta G, Hill V, Lanzman M, Balakrishnan I. $\beta$-lactam allergy: clinical implications and costs. Clin Mol Allergy. 2013;11(1):2. doi:10.1186/1476-7961-11-2

9. Dagli RJ, Sharma A. Polypharmacy: a global risk factor for elderly people. J Int Oral Heal JIOH. 2014;6(6):i-ii.

10. Blanca M, Torres MJ, García JJ, et al. Natural evolution of skin test sensitivity in patients allergic to beta-lactam antibiotics. J Allergy Clin Immunol. 1999;103(5 Pt 1):918-924. doi:10.1016/S00916749(99)70439-2

11. Gonzalez-Estrada A, Radojicic C. Penicillin allergy: a practical guide for clinicians. Cleve Clin J Med. 2015;82(5):295-300. doi:10.3949/ ccjm.82a.14111

12. Albin S, Agarwal S. Prevalence and characteristics of reported penicillin allergy in an urban outpatient adult population. Allergy Asthma Proc. 2014;35(6):489-494. doi:10.2500/aap.2014.35.3791

13. Macy E. Penicillin allergy. Curr Opin Allergy Clin Immunol. 2015;15 (4):308-313. doi:10.1097/ACI.0000000000000173

14. Moreno E, Laffond E, Muñoz-Bellido FJ, Gracia MT, Macías EM, Dávila I. Using $\beta$-lactam antibiotics in patients with a history of $\beta$ lactam allergy: current concepts. Polish Arch Intern Med. 2017;127 (7-8):540-549.

15. Pichichero ME, Zagursky R. Penicillin and cephalosporin allergy. Ann Allergy Asthma Immunol. 2014;112(5):404-412. doi:10.1016/j. anai.2014.02.005

16. Mattingly TJ, Fulton A, Lumish RA, et al. The cost of self-reported penicillin allergy: a systematic review. J Allergy Clin Immunol Pract. 2018;6(5):1649-1654.e4. doi:10.1016/j.jaip.2017.12.033

17. Li M, Krishna MT, Razaq S, Pillay D. A real-time prospective evaluation of clinical pharmaco-economic impact of diagnostic label of "penicillin allergy" in a UK teaching hospital. J Clin Pathol. 2014;67(12):1088-1092. doi:10.1136/jclinpath-2014-202438

18. de Bourcy CFA, Angel CJL, Vollmers C, Dekker CL, Davis MM, Quake SR. Phylogenetic analysis of the human antibody repertoire reveals quantitative signatures of immune senescence and aging. Proc Natl Acad Sci. 2017;114(5):1105-1110. doi:10.1073/pnas.1617959114

19. Sugahara H, Okai S, Odamaki T, et al. Decreased taxon-specific IgA response in relation to the changes of gut microbiota composition in the elderly. Front Microbiol. 2017;8:1-8. doi:10.3389/fmicb.2017.01757

20. Fernandez FJ, Jimenez-Rodriguez T, Soriano-Gomis V, RamosRincón JM, Lindo-Gutarra M, Castellanos LM. Allergy to betalactams antibiotics at the end-of-life care. J Allergy Clin Immunol. 2017;139(2):AB32. doi:10.1016/j.jaci.2016.12.161 
21. Baltes P, Smith J. New frontiers in the future of aging : from successful aging of the young old to the dilemmas of the fourth age. Gerontology. 2003;49(2):123-135. doi:10.1159/000067946

22. European Network on Drug Allergy. Protocolo de recogida de datos en los casos de sospecha de alergia a fármacos. Alergol Immunol Clin. 2001;16:48-53.

23. Brown SGA. Clinical features and severity grading of anaphylaxis. $J$ Allergy Clin Immunol. 2004;114(2):371-376. doi:10.1016/j. jaci.2004.04.029

24. Demoly P, Adkinson NF, Brockow K, et al. International consensus on drug allergy. Allergy. 2014;69(4):420-437. doi:10.1111/all.12350

25. Brockow K, Romano A, Blanca M, Ring J, Pichler W, Demoly P. General considerations for skin test procedures in the diagnosis of drug hypersensitivity. Allergy. 2002;57(1):45-51.

26. Torres MJ, Blanca M, Fernandez J, et al. Diagnosis of immediate allergic reactions to beta-lactam antibiotics. Allergy. 2003;58 (10):961-972. doi:10.1034/j.1398-9995.2003.00280.x

27. Amsler E, Soria A. Allergies aux bêtalactamines. Hypersensitivity reactions to beta-lactam antibiotics. La Rev Med Interne. 2017;38 (11):737-748. doi:10.1016/j.revmed.2017.06.020

28. Romano A, Gaeta F, Valluzzi RL, et al. IgE-mediated hypersensitivity to cephalosporins: cross-reactivity and tolerability of alternative cephalosporins. J Allergy Clin Immunol. 2015;136(3):685-691.e3. doi:10.1016/j.jaci.2015.03.012

29. Blanca M, Romano A, Torres MJ, et al. Update on the evaluation of hypersensitivity reactions to betalactams. Allergy Eur J Allergy Clin Immunol. 2009;64(2):183-193. doi:10.1111/all.2009.64.issue-2

30. Terrados S, Blanca M, Garcia J, et al. Nonimmediate reactions to betalactams: prevalence and role of the different penicillins. Allergy. 1995;50(7):563-567. doi:10.1111/all.1995.50.issue-7

31. Blanca-López N, Zapatero L, Alonso E, et al. Skin testing and drug provocation in the diagnosis of nonimmediate reactions to aminopenicillins in children. Allergy Eur J Allergy Clin Immunol. 2009;64 (2):229-233. doi:10.1111/all.2009.64.issue-2

32. Park MA, Solensky R, Khan DA, Castells MC, MacY EM, Lang DM. Patients with positive skin test results to penicillin should not undergo penicillin or amoxicillin challenge. J Allergy Clin Immunol. 2015;135(3):816-817. doi:10.1016/j.jaci.2014.08.045

33. Aberer W, Bircher A, Romano A, et al. Drug provocation testing in the diagnosis of drug hypersensitivity reactions: general considerations. Allergy. 2003;58(9):854-863. doi:10.1034/j.1398-9995.2003.00279.x

34. Patriarca G, D'Ambrosio C, Schiavino D, Nucera E. Allergy to betalactams: relationship between chemical structure and antigenicity of molecules. Invest Allergol Clin Immunol. 1999;9(4):215-221.

35. Lopez S, Blanca-Lopez N, Cornejo-Garcia JA, et al. Nonimmediate reactions to betalactams. Curr Opin Allergy Clin Immunol. 2007;7 (4):310-316. doi:10.1097/ACI.0b013e3281e209fe

36. Sage PT, Tan CL, Freeman GJ, Haigis M, Sharpe AH. Defective TFH cell function and increased TFR cells contribute to defective antibody production in aging. Cell Rep. 2015;12(2):163-171. doi:10.1016/j. celrep.2015.06.015

37. Minato N. T cell senescence and autoimmunity. In: Nakao K, Minato N, Uemoto S, editors. Innovative Medicine. Tokyo: Springer; 2015:119-128.

38. Scichilone N, Callari A, Augugliaro G, Marchese M, Togias A, Bellia V. The impact of age on prevalence of positive skin prick tests and specific IgE tests. Respir Med. 2011;105(5):651-658. doi:10.1016/j.rmed.2010.12.014

39. Antúnez C, Martín E, Cornejo-Garcia J, et al. Immediate hypersensitivity reactions to penicillins and other betalactams. Curr Pharm Des. 2006;12(26):3327-3333. doi:10.2174/138161206778194042

40. Fernandez TD, Mayorga C, Salas M, et al. Evolution of diagnostic approaches in betalactam hypersensitivity. Expert Rev Clin Pharmacol. 2017;10(6):671-683. doi:10.1080/17512433.2017. 1313110
41. Torres MJ, Romano A, Mayorga C, et al. Diagnostic evaluation of a large group of patients with immediate allergy to penicillins: the role of skin testing. Allergy. 2001;56(9):850-856. doi:10.1034/j.1398-9995.2001. 00089.x

42. Blanca-Lopez N, Perez-Alzate D, Ruano F, et al. Selective immediate responders to amoxicillin and clavulanic acid tolerate penicillin derivative administration after confirming the diagnosis. Allergy. 2015;70 (8):1013-1019. doi:10.1111/all.2015.70.issue-8

43. Blanca-Lopez N, Jimenez-Rodriguez TW, Somoza ML, et al. Allergic reactions to penicillins and cephalosporins: diagnosis, assessment of cross-reactivity and management. Expert Rev Clin Immunol. 2019;15 (7):707-721. doi:10.1080/1744666X.2019.1619548

44. Romano A, Torres MJ, Fernandez J, et al. Allergic reactions to ampicillin. Studies on the specificity and selectivity in subjects with immediate reactions. Clin Exp Allergy. 1997;27(12):1425-1431. doi:10.1111/cea.1997.27.issue-12

45. Romano A, Mayorga C, Torres MJ, et al. Immediate allergic reactions to cephalosporins: cross-reactivity and selective responses. J Allergy Clin Immunol. 2000;106(6):1177-1183. doi:10.1067/mai.2000.111147

46. Antunez C, Blanca-Lopez N, Torres MJ, et al. Immediate allergic reactions to cephalosporins: evaluation of cross-reactivity with a panel of penicillins and cephalosporins. J Allergy Clin Immunol. 2006;117(2):404-410. doi:10.1016/j.jaci.2005.10.032

47. Blanca M, Garcia J, Vega JM, et al. Anaphylaxis to penicillins after non-therapeutic exposure: an immunological investigation. Clin Exp Allergy. 1996;26(3):335-340. doi:10.1111/cea.1996.26.issue-3

48. Romano A, Gaeta F, Valluzzi RL, Zaffiro A, Caruso C, Quaratino D. Natural evolution of skin-test sensitivity in patients with IgE-mediated hypersensitivity to cephalosporins. Allergy. 2014;69 (6):806-809. doi:10.1111/all.2014.69.issue-6

49. Turner VM, Mabbott NA. Ageing adversely affects the migration and function of marginal zone B cells. Immunology. 2017;151 (3):349-362. doi:10.1111/imm.12737

50. Romano A, Blanca M, Torres MJ, et al. Diagnosis of nonimmediate reactions to beta-lactam antibiotics. Allergy Eur J Allergy Clin Immunol. 2004;59(11):1153-1160. doi:10.1111/j.1398-9995.2004. 00678.x

51. Demoly P, Romano A. Update on beta-lactam allergy diagnosis. Curr Allergy Asthma Rep. 2005;5(1):9-14. doi:10.1007/s11882005-0048-2

52. Macy E, Mangat R, Burchette RJ. Penicillin skin testing in advance of need: multiyear follow-up in 568 test result-negative subjects exposed to oral penicillins. J Allergy Clin Immunol. 2003;111 (5):1111-1115. doi:10.1067/mai.2003.1385

53. Brockow K. Triage strategies for clarifying reported betalactam allergy. J Allergy Clin Immunol Pract. 2019;7(3):1066-1067. doi:10.1016/j.jaip.2018.08.009

54. Blumenthal KG. The role of the clinical history in drug allergy prediction. J Allergy Clin Immunol Pract. 2018;6(1):149-150. doi:10.1016/j.jaip.2017.05.005

55. Moussa Y, Shuster J, Matte G, et al. De-labeling of $\beta$-lactam allergy reduces intraoperative time and optimizes choice in antibiotic prophylaxis. Surgery. 2018;164:117-123. doi:10.1016/j.surg.2018.03.004

56. Centers for Disease Control and Prevention. Is it really a penicillin allergy? [Internet]. 2016. Available from: http://www.cdc.gov/getsmart/ week/downloads/getsmart-penicillin-factsheet.pdf. Accessed December 4, 2019.

57. Bourke J, Pavlos R, James I, Phillips E. Improving the effectiveness of penicillin allergy de-labeling. J Allergy Clin Immunol Pract. 2015;3(3):365-34.e1. doi:10.1016/j.jaip.2014.11.002

58. Gerace KS, Phillips E. Penicillin allergy label persists despite negative testing. J Allergy Clin Immunol Pract. 2015;3(5):815-816. doi:10.1016/j.jaip.2015.05.019 
59. Trubiano J, Phillips E. Antimicrobial stewardship's new weapon? A review of antibiotic allergy and pathways to "de-labeling.". Curr Opin Infect Dis. 2013;26(6):526-537. doi:10.1097/QCO.0000 000000000006
60. Hjortlund J, Mortz CG, Skov PS, Bindslev-Jensen C. Diagnosis of penicillin allergy revisited: the value of case history, skin testing, specific IgE and prolonged challenge. Allergy. 2013;68 (8):1057-1064. doi:10.1111/all.2013.68.issue-8

\section{Publish your work in this journal}

The Journal of Asthma and Allergy is an international, peer-reviewed open-access journal publishing original research, reports, editorials and commentaries on the following topics: Asthma; Pulmonary physiology; Asthma related clinical health; Clinical immunology and the immunological basis of disease; Pharmacological interventions and

Submit your manuscript here: https://www.dovepress.com/journal-of-asthma-and-allergy-journa new therapies. The manuscript management system is completely online and includes a very quick and fair peer-review system, which is all easy to use. Visit http://www.dovepress.com/testimonials.php to read real quotes from published authors. 